05

\title{
Модуль упругости, поверхностного натяжения, адгезии, идеальная и реальная прочность твердых тел
}

\author{
(C) Е.Ф. Кустов, ${ }^{1}$ М.Е. Кустов, ${ }^{2}$ В.А. Антонов ${ }^{1,}$ \\ ${ }^{1}$ Национальный исследовательский университет (МЭИ), \\ 112250 Москва, Россия \\ ${ }^{2}$ Научно-исследовательский институт технического стекла, \\ 117218 Москва, Россия \\ ๑ e-mail: Antonov913@yandex.ru
}

(Поступило в Редакцию 2 сентября 2016 г.)

Получены формулы поверхностного натяжения, энергии адгезии, модуля упругости и прочности твердых тел, в зависимости от плотности атомов. Теоретические значения этих величин подтверждаются экспериментальными значениями на большом массиве металлов, диэлектриков, полупроводников.

DOI: 10.21883/JTF.2017.04.44317.2026

\section{Введение}

Поверхностное натяжение представляет собой основную термодинамическую характеристику поверхностного слоя жидкостей и твердых тел на границе с различными фазами (газами, жидкостями, твердыми телами).

Поверхностное натяжение определяет такие важные для физики величины, как капиллярное давление жидкости под искривленной поверхностью по уравнению Лапласа; краевой угол смачивания, образуемый жидкостью возле поверхности твердого тела по уравнению Юнга $[1,2]$. Кроме того, оно определяет давление насыщенного пара над искривленной поверхностью жидкости по уравнению Кельвина, адсорбцию веществ, самопроизвольно концентрирующихся на поверхности раздела фаз по уравнению Гиббса. Поверхностное натяжение определяет эффекты, связанные с электрическими полями на поверхности. Это электрокапиллярный эффект по уравнению Липпмана и дифференциальная емкость двойного электрического слоя и т. п. Оно же определяет внешние формы веществ: равновесную форму (огранку) кристаллов, работу образования критического зародыша при образовании новой фазы, а также идеальную и реальную прочности твердых тел. Из анализа свойств двойного слоя атомов можно получить формулу модуля упругости, зависящего от плотности атомов в степени $N_{V}^{4 / 3}$ и параметра сил отталкивания. Теоретические значения модуля упругости $E$ подтверждаются экспериментальными значениями на большом массиве металлов, диэлектриков, полупроводников.

Эти рассмотрения позволяют получить формулу для механической прочности упругих тел, которые зависят от произведения модуля упругости (модуль Юнга) и поверхностного натяжения $E_{\gamma}$.

\section{Сила и энергия взаимодействия двойного слоя атомов}

Для силы и энергии взаимодействия между атомами в модели двойного слоя была получена [3] следующая формула:

$$
\begin{aligned}
& F=\frac{B}{r_{0}^{2}}\left(-\frac{1}{(1+x)^{n+1}}+\frac{1}{(1+x)^{2}}\right), \\
& W=\frac{B}{r_{0}}\left(-\frac{1}{n(x+1)^{n}}-\frac{1}{(x+1)}\right),
\end{aligned}
$$

где $x=\varepsilon-1$ и $\varepsilon=\frac{r}{r_{0}}$ - деформация относительно равновесного расстояния $r_{0}, B$ - параметр взаимодействия между атомами, $n$ - параметр теории, определяющей силы отталкивания при взаимодействии атомов в твердом теле.

Модуль упругости Юнга $E$ можно найти из значения производной силы по расстоянию, умноженной на количество атомов $N_{S}$ на единице площади двойного слоя:

$$
E=\left.N_{S} \frac{d F}{d x}\right|_{x,(W=0)} .
$$

Состояние упругости определяется не условием равновесия, где $F=0, x=0$ и $r=r_{0}$, а условием $r<r_{0}$ и $x<0$, где силы отталкивания превалируют над силами притяжения.

Модуль упругости, определенный в области деформаций $r<r_{0}$ и $x<0$, равен

$$
E=2.3 \cdot 10^{-29} N_{V}^{4 / 3}\left((n+1) n^{\frac{n+2}{n-1}}-2 n^{\frac{3}{n-1}}\right),
$$

где $N_{V}$ - объемная плотность атомов.

Объемная плотность атомов равна

$$
N_{V}=-\frac{\rho}{m}(\mathrm{~N} / \mathrm{m})
$$

где $\rho-$ плотность вещества, $M-$ вес молекулы в единицах атомной массы, $m=1.66 \cdot 10^{-27} \mathrm{~kg}$ - единица атомной массы.

Аналогично формула энергии поверхностного натяжения через поверхностную атомную плотность имеет вид

$$
\gamma=2.31 \cdot 10^{-28} N_{S}^{3 / 2} \frac{n-1}{2 n},
$$

где $N_{S}-$ поверхностная атомная плотность, $\mathrm{m}^{-2}$. 


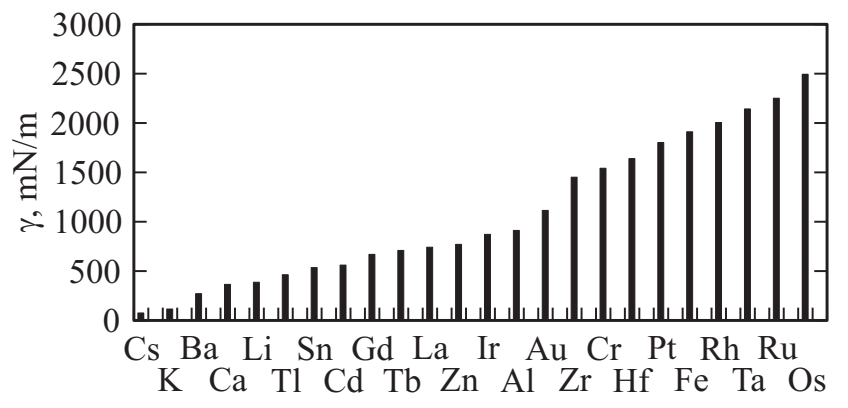

Рис. 1. Диаграмма поверхностного натяжения расплавов металлов.

\section{Поверхностное натяжение элементов}

Значения величины поверхностного натяжения элементов периодической системы, рассчитанные по выведенной формуле (5), приведены на рис. 1. Значения параметра отталкивания $n$ были получены из сравнения теоретической и экспериментальных значений поверхностного натяжения. Следует отметить, что изменение этого параметра на массиве элементов находится в пределе $n=1-2$, в то время как значения поверхностного натяжения меняются на 3-4 порядка, отслеживая изменение атомной поверхностной плотности, как это предсказывает формула (5).

\section{Адгезия твердых тел}

Равновесная работа адгезии $A$ определяется убылью свободной поверхностной энергии

$$
A=\gamma_{c}+\gamma_{a}-\gamma_{c a},
$$

где $\gamma_{c}, \gamma_{a}$ - энергии поверхностного натяжения субстрата $(c)$ и адгезива $(a)$ до адгезии и $\gamma_{c a}-$ при адгезии субстрата и адгезива [4].

Подставляя в (6) формулу (5), для субстрата, адгезива и слоя субстрат-адгезив получим формулу энергии адгезии субстрата и адгезива

$$
\begin{aligned}
A=2.31 \cdot 10^{-28} & {\left[N_{S_{c}}^{3 / 2} \frac{n_{c}-1}{2 n_{c}}\right.} \\
& \left.+N_{S_{a}}^{3 / 2} \frac{n_{c}-1}{2 n_{a}}-N_{S_{c a}}^{3 / 2} \frac{n_{c a}-1}{2 n_{c a}}\right] .
\end{aligned}
$$

Для атомной поверхностной плотности субстрата и адгезива формулу (4) можно представить следующим образом:

$$
N_{S_{c}}^{3 / 2}=\frac{\rho_{c}}{M_{c} m}, N_{S_{a}}^{3 / 2}=\frac{\rho_{a}}{M_{a} m}, N_{S_{c a}}^{3 / 2}=\frac{\rho_{c}+\rho_{a}}{\left(M_{c}+M_{a}\right) m}
$$

Таблица 1. Энергия адгезии некоторых материалов к пленкам $\mathrm{In}_{2} \mathrm{O}_{3}, \mathrm{In}, \mathrm{SnO}_{2}, \mathrm{Sn}, \mathrm{H}_{2} \mathrm{O}$

\begin{tabular}{l|c|c|c|c|c}
\hline \multirow{2}{*}{ Субстрат } & \multicolumn{5}{|c}{ Энергия адгезии $A, \mathrm{~J} / \mathrm{m}^{2}$} \\
\cline { 2 - 6 } & $\mathrm{In}_{2} \mathrm{O}_{3}$ & $\mathrm{In}$ & $\mathrm{SnO}_{2}$ & $\mathrm{Sn}$ & $\mathrm{H}_{2} \mathrm{O}$ \\
\hline $\mathrm{SiO}_{2}$ & 0.889 & 1.884 & 0.920 & 1.838 & 0.694 \\
$\mathrm{Al}_{2} \mathrm{O}_{3}$ & 1.759 & 3.727 & 1.820 & 3.638 & 1.373 \\
$\mathrm{BeO}$ & 1.468 & 3.110 & 1.518 & 3.036 & 1.146 \\
$\mathrm{MgO}$ & 1.367 & 2.896 & 1.414 & 2.827 & 1.067 \\
$\mathrm{ZrO}_{2}$ & 1.283 & 2.718 & 1.327 & 2.653 & 1.001 \\
$\mathrm{ThO}_{2}$ & 1.155 & 2.447 & 1.195 & 2.388 & 0.901 \\
$\mathrm{C}_{\text {текло 1 }}$ & 0.128 & 0.271 & 0.132 & 0.264 & 0.100 \\
Стекло 2 & 0.131 & 0.277 & 0.135 & 0.270 & 0.102 \\
Стекло 3 & 0.139 & 0.295 & 0.144 & 0.288 & 0.109 \\
Стекло 4 & 0.139 & 0.295 & 0.144 & 0.288 & 0.109 \\
Стекло 5 & 0.263 & 0.557 & 0.272 & 0.543 & 0.205 \\
Стекло 6 & 0.248 & 0.525 & 0.256 & 0.513 & 0.194 \\
Стекло & 0.232 & 0.492 & 0.240 & 0.480 & 0.181 \\
Пирекс & & & & & $0.146^{*}$
\end{tabular}

Примечани е. * Работа адгезии, измеренная экспериментально [5].

Тогда энергия адгезии будет

$$
\begin{aligned}
A=2.31 \cdot 10^{-29}\left[\frac{\rho_{c}}{M_{c} m}\right. & \frac{n_{c}-1}{2 n_{c}}+\frac{\rho_{a}}{M_{a} m} \frac{n_{a}-1}{2 n_{a}} \\
& \left.-\frac{\rho_{c}+\rho_{a}}{\left(M_{c}+M_{a}\right) m} \frac{n_{c a}-1}{2 n_{c a}}\right] .
\end{aligned}
$$

Если учесть величину единицы атомной массы $m=$ $=1.66 \cdot 10^{-27} \mathrm{~kg}$, эта формула будет иметь следующий вид:

$$
\begin{aligned}
A=1.38\left[\frac{\rho_{c}}{M_{c}}\right. & \frac{n_{c}-1}{2 n_{c}}+\frac{\rho_{a}}{M_{a}} \frac{n_{a}-1}{2 n_{a}} \\
& \left.-\frac{\rho_{c}+\rho_{a}}{\left(M_{c}+M_{a}\right)} \frac{n_{c a}-1}{2 n_{c a}}\right],
\end{aligned}
$$

где $\rho$ - плотность вещества, $\mathrm{kg} / \mathrm{m}^{3}, M-$ вес молекулы в единицах атомной массы.

Второе приближение для энергии адгезии можно получить через среднегеометрическое значение энергии поверхностного натяжения субстрата и адгезива [5]

$$
A=2 \sqrt{\gamma_{c} \gamma_{a}} \text {. }
$$

Подставляя формулу (5) в (9), для энергии адгезии получим

$$
A=2.31 \cdot 10^{-28}\left[N_{S_{c}}^{3 / 4} N_{S_{a}}^{3 / 4} \sqrt{\frac{n_{c}-1}{n_{c}} \frac{n_{a}-1}{n_{a}}}\right] .
$$

Эта же формула через атомные плотности субстрата и адгезива будет иметь следующий вид:

$$
A=0.138 \sqrt{\frac{\rho_{c}}{M_{c}} \frac{\rho_{a}}{M_{a}} \frac{n_{c}-1}{n_{c}} \frac{n_{a}-1}{n_{a}}}\left(\mathrm{~J} / \mathrm{m}^{2}\right) .
$$


Таблица 2. Спецификация стекол

\begin{tabular}{l|l}
\hline Стекло 1 & Кварцевое непрозрачное \\
\hline Стекло 2 & Кварцевое прозрачное \\
\hline Стекло 3 & Электровакуумное \\
\hline Стекло 4 & Электроизоляционное \\
\hline Стекло 5 & Иенское крон \\
\hline Стекло 6 & Иенское флинт
\end{tabular}

Непосредственное измерение энергии адгезии является трудным в реализации экспериментом. Поэтому энергия адгезии, рассчитанная теоретически по формулам (8) или (11), может служить ориентиром для экспериментаторов, по которому можно проводить сравнительный анализ изменения энергии адгезии для различных материалов. В табл. 1 даны эти величины для некоторых проводящих оптически прозрачных пленок, применяющихся для изделий конструктивной оптики. В табл. 2 приведена спецификация стекол, для которых определялась энергия адгезии.

Обычно измеряют адгезионную прочность по силе отрыва пленки. Сила отрыва пленки зависит от геометрических размеров пленки, скорости отрыва и т.п., а также процессов когезии, т. е. силы отрыва слоев внутри адгезива или субстрата.

Работу адгезии жидкости к поверхности твердых тел можно измерить экспериментально по краевому углу смачивания $\theta$ жидкости с поверхностным натяжением $\gamma_{a}[6]$

$$
A=\gamma_{a}(1+\cos \theta) \text {. }
$$

По формуле (13) и формуле (9) можно определить угол смачивания

$$
1+\cos \theta=2 \sqrt{\frac{\gamma_{c}}{\gamma_{a}}}=2 \frac{N_{S_{c} / 4}^{3 / 4}}{N_{S_{a}}^{3 / 4}} \sqrt{\frac{n_{a}\left(n_{c}-1\right)}{n_{c}\left(n_{a}-1\right)}} .
$$

В табл. 1 приведена также величина энергии адгезии, полученная по измерению угла смачивания $\mathrm{H}_{2} \mathrm{O}$ поверхности пирекса.

\section{Формула модуля упругости и эксперимент}

Модуль упругости и параметр сил отталкивания элементов периодической системы, рассчитанный по формуле (3), представлен в табл. 3 и на рис. 2 и 3 одинаковой последовательностью элементов.

Разница теоретически рассчитанного и экспериментального значения модуля упругости составляет десятые доли процента.

В табл. 4 и 5, на диаграммах рис. 4 и 5 приведены модули упругости неорганических веществ и некоторых

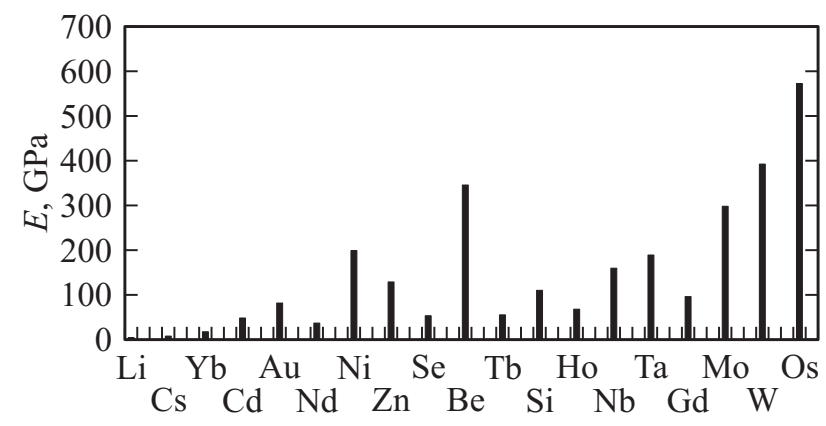

Рис. 2. Диаграмма модуля упругости Е некоторых элементов периодической системы.

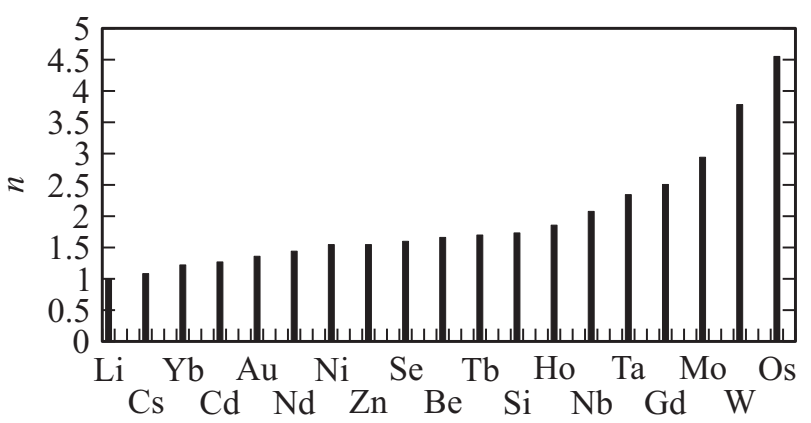

Pис. 3. Диаграмма параметра сил отталкивания $\mathrm{n}$ некоторых элементов периодической системы.

стекол, рассчитанные теоретически по вышеприведенным формулам, а также экспериментальные значения для некоторых веществ.

Экспериментальные значения модуля упругости брались из работы [13].

\section{Модуль прочности твердых тел}

Условие разрушения материалов определяется условиями изменения энергии тела при возникновении и распространении трещин или других дефектов.

Можно рассматривать идеальную $P_{\text {id }}$ и реальную $P_{\text {real }}$ прочности $\left(\mathrm{N} / \mathrm{m}^{2}\right)$ твердых тел. Идеальная $P_{\text {id }}$ и реальная $P_{\text {real }}$ прочности твердых тел определяются уравнениями Поляни-Смекала и Гриффитса:

$$
P_{\text {id }}=\sqrt{\frac{2 E \gamma}{\delta}}, \quad P_{\text {real }}=\sqrt{\frac{2 E \gamma}{\pi l}},
$$

где $E-$ модуль Юнга, $\gamma-$ поверхностное натяжение, $\delta$ - межатомное расстояние, $l$ - длина зародышевой трещины, которая при напряжении $P_{\text {real }}$ начинает самопроизвольно расти.

Во всех этих теориях используется произведение модуля Юнга на поверхностное натяжение. Можно использовать формулы модуля упругости (3) и поверхностного 


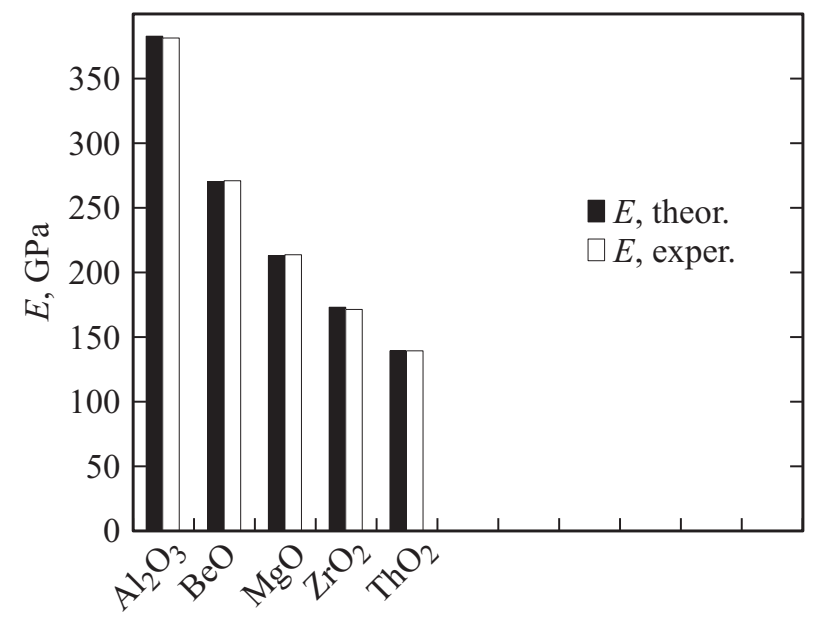

Рис. 4. Диаграмма модуля упругости неорганических веществ.

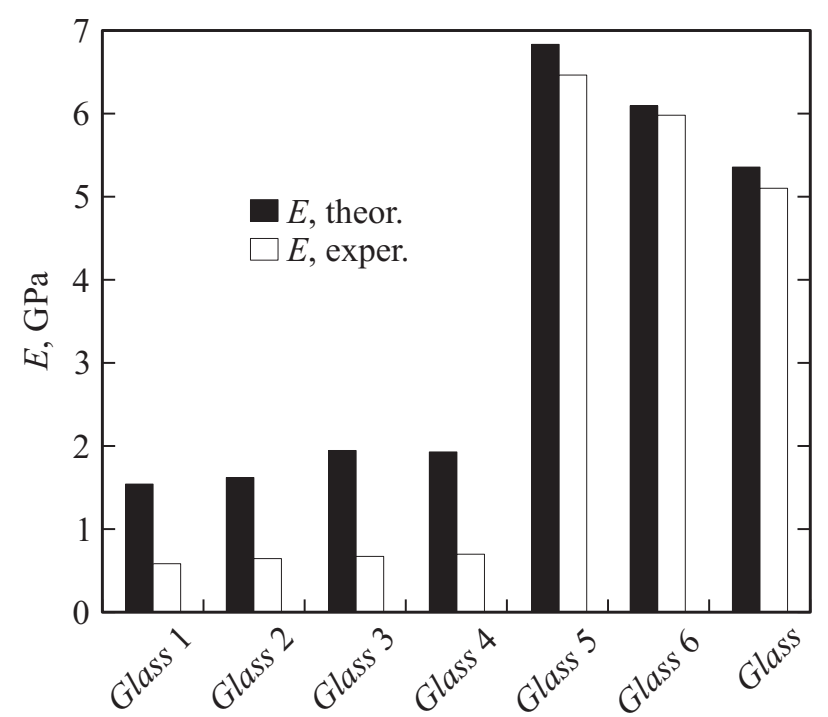

Pис. 5. Диаграмма модуля упругости стекол.

натяжения (5), и произведение $E \gamma$ будет равно:

$$
E \gamma=5.31 \cdot 10^{-57} N_{V}^{7 / 3} \frac{n-1}{2 n}\left((n+1) n^{\frac{n+2}{n-1}}-2 n^{\frac{3}{n-1}}\right)
$$

Подставляя это произведение в формулы (14), получим формулы идеальной и реальной прочностей твердых тел:

$$
P_{\text {id }}=7.29 \cdot 10^{-29} N_{V}^{7 / 6} \sqrt{\frac{n-1}{n}\left((n+1) n^{\frac{n+2}{n-1}}-2 n^{\frac{3}{n-1}}\right) \frac{1}{\delta}},
$$

$P_{\text {real }}=7.29 \cdot 10^{-29} N_{V}^{7 / 6} \sqrt{\frac{n-1}{n}\left((n+1) n^{\frac{n+2}{n-1}}-2 n^{\frac{3}{n-1}}\right) \frac{1}{\pi l}}$.

В формуле (16) можно ввести упрощение $\delta=l^{\prime} N_{v}^{-1 / 3}$, или $\pi l=l^{\prime} N_{V}^{-1 / 3}$, где $l^{\prime}$ - длина трещины в относительных единицах расстояния между атомами.
Внося это упрощение в формулу идеальной и реальной прочностей, получим

$$
\begin{aligned}
P= & 7.29 \cdot 10^{-29} N_{V}^{4 / 3} \\
& \times \sqrt{\frac{n-1}{n}\left((n+1) n^{\frac{n+2}{n-1}}-2 n^{\frac{3}{n-1}}\right)}(\mathrm{Pa}) .
\end{aligned}
$$

В зависимости от объемной плотности атомов $N_{V}$ предел прочности определяется как $N_{V}^{4 / 3}$, также как модуль упругости.

Для получения точного значения предела прочности необходимо вводить относительную длину трещины $l^{\prime}$ как параметр и определять ее значение из сравнения экспериментальных и теоретических значений предела прочности. Однако можно использовать формулы (16) и (17) для определения фактора прочности $\delta$, как отклонения реальной прочности веществ от идеальной:

$$
\delta=\frac{P\left(l^{\prime}\right)}{P\left(l^{\prime}=1\right)}=\frac{1}{\sqrt{l^{\prime}}} .
$$

Фактор прочности меняется от единицы, $\delta=1$, когда реальная прочность равна идеальной, до $\delta=0, l^{\prime} \gg 1$,

Таблица 3. Модуль упругости и параметр сил отталкивания элементов периодической системы

\begin{tabular}{c|c|c||c|c|c}
\hline Элемент & $E, \mathrm{GPa}$ & $n$ & Элемент & $E, \mathrm{GPa}$ & $n$ \\
\hline $\mathrm{Cs}$ & 1.76801 & 1.0802 & $\mathrm{Gd}$ & 97.82058 & 2.52 \\
$\mathrm{Rb}$ & 2.458781 & 1.081 & $\mathrm{Pr}$ & 98.50032 & 2.8 \\
$\mathrm{Li}$ & 5.02923 & 1.0223 & $\mathrm{Dy}$ & 98.75342 & 2.45 \\
$\mathrm{Tl}$ & 7.92622 & 1.053 & $\mathrm{Si}$ & 109.955 & 1.73 \\
$\mathrm{In}$ & 10.55944 & 1.063 & $\mathrm{Ti}$ & 110.5316 & 1.575 \\
$\mathrm{~Pb}$ & 17.98408 & 1.142 & $\mathrm{Er}$ & 114.9799 & 2.785 \\
$\mathrm{Yb}$ & 18.02245 & 1.232 & $\mathrm{Pd}$ & 125.0918 & 1.487 \\
$\mathrm{Ca}$ & 26.03343 & 1.4 & $\mathrm{Cu}$ & 129.9451 & 1.342 \\
$\mathrm{Bi}$ & 32.00141 & 1.375 & $\mathrm{Zn}$ & 130.5869 & 1.55 \\
$\mathrm{La}$ & 38.38118 & 1.5348 & $\mathrm{Hf}$ & 150.6995 & 2.48 \\
$\mathrm{Nd}$ & 38.39719 & 1.45 & $\mathrm{Nb}$ & 160.4527 & 2.08 \\
$\mathrm{Ce}$ & 44.02304 & 1.55 & $\mathrm{~V}$ & 170.9378 & 1.68 \\
$\mathrm{Te}$ & 44.73772 & 1.55 & $\mathrm{Pt}$ & 174.791 & 1.83 \\
$\mathrm{Mg}$ & 44.86112 & 1.278 & $\mathrm{Ta}$ & 190.3775 & 2.35 \\
$\mathrm{Cd}$ & 49.95116 & 1.282 & $\mathrm{Mn}$ & 199.7896 & 1.672 \\
$\mathrm{Se}$ & 54.52143 & 1.6 & $\mathrm{Ni}$ & 200.0146 & 1.54 \\
$\mathrm{Sn}$ & 54.91102 & 1.73 & $\mathrm{Fe}$ & 200.1158 & 1.625 \\
$\mathrm{Sm}$ & 55.00902 & 1.708 & $\mathrm{Co}$ & 206.0308 & 1.569 \\
$\mathrm{~Tb}$ & 57.40401 & 1.7 & $\mathrm{U}$ & 210.1488 & 3 \\
$\mathrm{Y}$ & 65.93305 & 1.925 & $\mathrm{Mo}$ & 300.1759 & 2.94 \\
$\mathrm{Ho}$ & 67.5904 & 1.85 & $\mathrm{Cr}$ & 314.6545 & 2.265 \\
$\mathrm{Al}$ & 69.51709 & 1.275 & $\mathrm{Be}$ & 345.4163 & 1.665 \\
$\mathrm{Sb}$ & 77.40375 & 1.98 & $\mathrm{Rh}$ & 384.242 & 3.15 \\
$\mathrm{Th}$ & 79.26929 & 2.2 & $\mathrm{~W}$ & 394.4927 & 3.8 \\
$\mathrm{Ag}$ & 80.68181 & 1.35 & $\mathrm{Re}$ & 474.2556 & 4.1 \\
$\mathrm{Ge}$ & 81.51387 & 1.6 & $\mathrm{Ru}$ & 500.8337 & 3.9 \\
$\mathrm{Au}$ & 83.02654 & 1.36 & $\mathrm{Os}$ & 574.9851 & 4.56 \\
$\mathrm{Zr}$ & 96.90856 & 1.82 & $\mathrm{Ir}$ & 589.8954 & 4.727 \\
& & & & & \\
\end{tabular}

Примечание. Экспериментальные значения модуля упругости брались из следующих справочников и научных работ [7-12]. 
когда отклонение реальной прочности от идеальной существенно. В табл. 6 и 7 приведены факторы прочности некоторых элементов периодической системы и неорганических веществ, рассчитанные по формуле (19).

Таблица 4. Модуль упругости неорганических веществ и стекол

\begin{tabular}{l|c|c|c|c}
\hline $\mathrm{Beщество}$ & $\begin{array}{c}N_{V} \cdot 10^{28} \\
\mathrm{~m}^{-3}\end{array}$ & $\begin{array}{c}\text { Плотность, } \\
\mathrm{kg} / \mathrm{m}^{3}\end{array}$ & $n$ & $\begin{array}{c}E, \mathrm{GPa} \\
\text { (теоретические) }\end{array}$ \\
\hline $\mathrm{CuCl}$ & 4.5 & 3700 & 1.21 & 37.9 \\
$\mathrm{CuBr}$ & 3.96 & 4720 & 1.21 & 32.0 \\
$\mathrm{CuI}$ & 3.57 & 5650 & 1.21 & 27.9 \\
$\mathrm{AgI}$ & 2.91 & 5670 & 1.21 & 21.2 \\
$\mathrm{BeS}$ & 6.92 & 2360 & 1.21 & 67.3 \\
$\mathrm{MnS}$ & 5.53 & 3990 & 1.21 & 49.8 \\
$\mathrm{MnSe}$ & 4.99 & 5550 & 1.21 & 43.5 \\
$\mathrm{ZnS}$ & 5.06 & 4090 & 1.21 & 44.3 \\
$\mathrm{ZnSe}$ & 4.52 & 5420 & 1.21 & 38.2 \\
$\mathrm{ZnTe}$ & 3.96 & 6340 & 1.21 & 31.9 \\
$\mathrm{CdTe}$ & 2.94 & 5860 & 1.21 & 21.5 \\
$\mathrm{HgS}$ & 4 & 7730 & 1.21 & 32.4 \\
$\mathrm{HgSe}$ & 3.56 & 8270 & 1.21 & 27.8 \\
$\mathrm{HgTe}$ & 2.97 & 8090 & 1.21 & 21.8 \\
$\mathrm{AlP}$ & 4.99 & 2400 & 1.21 & 43.5 \\
$\mathrm{AlAs}$ & 4.37 & 3700 & 1.21 & 36.5 \\
$\mathrm{AlSb}$ & 3.48 & 4300 & 1.21 & 26.9 \\
$\mathrm{GaP}$ & 4.95 & 4140 & 1.21 & 43.1 \\
$\mathrm{GaAs}$ & 4.46 & 5350 & 1.21 & 37.4 \\
$\mathrm{GaSb}$ & 3.53 & 5610 & 1.21 & 27.4 \\
$\mathrm{InP}$ & 3.96 & 4790 & 1.21 & 31.9 \\
$\mathrm{InAs}$ & 3.61 & 5680 & 1.21 & 28.2 \\
$\mathrm{InSb}$ & 2.94 & 5780 & 1.21 & 21.5 \\
$\mathrm{BP}$ & 8.33 & 2890 & 1.21 & 86.2 \\
$\mathrm{SiC}$ & 9.65 & 3210 & 1.21 & 104.7 \\
$\mathrm{SiO} 2$ & 7.97 & 2650 & 1.21 & 81.2 \\
& & & & \\
& & &
\end{tabular}

Таблица 5. Сравнение рассчитанных значений модуля упругости неоганических веществ и стекол со значениями, полученными экспериментально

\begin{tabular}{l|c|c|c|c|c}
\hline Вещество & $\begin{array}{c}N_{V} \cdot 10^{28}, \\
\mathrm{~m}^{-3}\end{array}$ & $\begin{array}{c}\text { Плотность, } \\
\mathrm{kg} / \mathrm{m}^{3}\end{array}$ & $n$ & $\begin{array}{c}E, \mathrm{GPa} \\
\text { (теорети- } \\
\text { ческие) }\end{array}$ & $\begin{array}{c}E, \mathrm{GPa} \\
(\text { эксперимен- } \\
\text { тальные) }\end{array}$ \\
\hline $\mathrm{Al}_{2} \mathrm{O}_{3}$ & 1.18 & 3990 & 1.85 & 382.5 & 382 \\
$\mathrm{BeO}$ & 14.5 & 3020 & 1.35 & 271.0 & 272 \\
$\mathrm{MgO}$ & 10.7 & 3580 & 1.44 & 213.1 & 214 \\
$\mathrm{ZrO}_{2}$ & 8.21 & 5600 & 1.54 & 173.3 & 172 \\
$\mathrm{ThO}_{2}$ & 8.32 & 9690 & 1.39 & 139.5 & 140 \\
$\mathrm{C}_{2}$ 1 & 5.74 & 2100 & 1.01 & 1.5 & 0.6 \\
Стекло 2 & 6.01 & 2200 & 1.01 & 1.6 & 0.65 \\
Стекло 3 & 6.83 & 2500 & 1.01 & 1.9 & 0.65 \\
Стекло 4 & 6.83 & 2500 & 1.01 & 1.9 & 0.7 \\
Стекло 5 & 6.83 & 2500 & 1.018 & 6.8 & 6.5 \\
Стекло 6 & 6.83 & 2500 & 1.016 & 6.1 & 6 \\
Стекло & 6.83 & 2500 & 1.014 & 5.3 & 5.1
\end{tabular}

Примечание. Спецификация стекол 1-6 предсталена в табл. 2. Типы стекол на диаграммах соответствуют табл. 2.
Таблица 6. Фактор прочности элементов периодической системы

\begin{tabular}{l|r|c|c|r|c}
\hline Элемент & $l^{\prime}, \mathrm{m}$ & $\delta, \%$ & Элемент & $l^{\prime}, \mathrm{m}$ & $\delta, \%$ \\
\hline $\mathrm{Cs}$ & 1 & 96.83 & $\mathrm{Dy}$ & 2803 & 1.89 \\
$\mathrm{Li}$ & 44 & 15.14 & $\mathrm{Pr}$ & 12347 & 0.90 \\
$\mathrm{Tl}$ & 190700 & 0.23 & $\mathrm{Ti}$ & 6578 & 1.23 \\
$\mathrm{Yb}$ & 3838 & 1.61 & $\mathrm{Er}$ & 2276 & 2.10 \\
$\mathrm{~Pb}$ & 165564 & 0.25 & $\mathrm{Pd}$ & 15566 & 0.80 \\
$\mathrm{Ca}$ & 8213 & 1.10 & $\mathrm{Cu}$ & 17915 & 0.75 \\
$\mathrm{Bi}$ & 117162 & 0.29 & $\mathrm{Zn}$ & 37884 & 0.51 \\
$\mathrm{La}$ & 3086 & 1.80 & $\mathrm{Hf}$ & 821 & 3.49 \\
$\mathrm{Nd}$ & 2042 & 2.21 & $\mathrm{Si}$ & 705 & 3.77 \\
$\mathrm{Te}$ & 694738 & 0.12 & $\mathrm{Nb}$ & 7476 & 1.16 \\
$\mathrm{Ce}$ & 6102 & 1.28 & $\mathrm{~V}$ & 18101 & 0.74 \\
$\mathrm{Mg}$ & 8756 & 1.07 & $\mathrm{Pt}$ & 38075 & 0.51 \\
$\mathrm{Cd}$ & 25239 & 0.63 & $\mathrm{Ta}$ & 2736 & 1.91 \\
$\mathrm{Sn}$ & 95722 & 0.32 & $\mathrm{Fe}$ & 15126 & 0.81 \\
$\mathrm{Sm}$ & 5649 & 1.33 & $\mathrm{Ni}$ & 8791 & 1.07 \\
$\mathrm{Y}$ & 1177 & 2.91 & $\mathrm{Co}$ & 24981 & 0.63 \\
$\mathrm{Ho}$ & 1743 & 2.40 & $\mathrm{U}$ & 6988 & 1.20 \\
$\mathrm{Al}$ & 112180 & 0.30 & $\mathrm{Mo}$ & 2925 & 1.85 \\
$\mathrm{Sb}$ & 2194387 & 0.07 & $\mathrm{Cr}$ & 21707 & 0.68 \\
$\mathrm{Ag}$ & 16006 & 0.79 & $\mathrm{Be}$ & 185315 & 0.23 \\
$\mathrm{Th}$ & 2655 & 1.94 & $\mathrm{Rh}$ & 12438 & 0.90 \\
$\mathrm{Ge}$ & 46949 & 0.46 & $\mathrm{~W}$ & 6786 & 1.21 \\
$\mathrm{Au}$ & 14455 & 0.83 & $\mathrm{Re}$ & 8987 & 1.05 \\
$\mathrm{Zr}$ & 4690 & 1.46 & $\mathrm{Ru}$ & 10617 & 0.97 \\
$\mathrm{Gd}$ & 1066 & 3.06 & $\mathrm{Ir}$ & 18386 & 0.74 \\
& & & & &
\end{tabular}

Таблица 7. Фактор прочности неорганических веществ

\begin{tabular}{l|r|r}
\hline Неорганическое вещество & $l^{\prime}, \mathrm{m}$ & $\delta, \%$ \\
\hline $\mathrm{Al}_{2} \mathrm{O}_{3}$ & 346199 & 0.17 \\
$\mathrm{BeO}$ & 1123322 & 0.09 \\
$\mathrm{MgO}$ & 793819 & 0.11 \\
$\mathrm{ZrO}_{2}$ & 228215 & 0.21 \\
$\mathrm{ThO}_{2}$ & 327470 & 0.17 \\
Стекло 1 & 243 & 6.42 \\
Стекло 2 & 122 & 9.05 \\
Стекло 3 & 247 & 6.36
\end{tabular}

Исходя из анализа этих данных, следует выделить щелочные элементы $\mathrm{Cs}, \mathrm{Li}$, для которых $\delta \approx 100$ и $\approx 15 \%$ соответственно.

Из неорганических материалов максимальный предел прочности имеют стекла $\delta \approx 6-9 \%$.

\section{Заключение}

Теоретические значения поверхностного натяжения, энергии адгезии, модуля упругости и прочности твердых тел, полученные в настоящей работе, подтверждаются экспериментальными значениями на большом массиве металлов, диэлектриков, полупроводников. Установлено, 
что энергия поверхностного натяжения, модули упругости и прочности твердых тел зависят от поверхностной и объемной концентрации атомов, как $N_{S}^{3 / 2}$ и $N_{v}^{4 / 3}$.

\section{Список литературы}

[1] Гиббс Джс.В. Термодинамика. Статистическая механика. М.: Наука, 1982. 584 c.

[2] Абрамзон А.А. Поверхностно-активные вещества. Л.: Химия, 1981. $200 \mathrm{c}$.

[3] Кустов М.Ф., Кустов Е.Ф. Мирочниченко А.Ю., Шеметова В.К. // Вестник МЭИ. 2013. № 5. С. 162-168.

[4] Физическая энциклопедия в пяти томах / Под ред. А.М. Прохорова. Т. 1. М.: Советская энциклопедия, 1988. $503 \mathrm{c}$.

[5] Зимон А.Д. Адгезия жидкости и смачивание. М.: Химия, 1974. $413 \mathrm{c}$.

[6] Зимон А.Д. Адгезия пленок и покрытий. М.: Химия, 1977. $352 \mathrm{c}$.

[7] Металловедение и термическая обработка стали. Справочник. Т. 1. М.: Изд-во по черной и цветной металлургии, 1961. $1204 \mathrm{c}$.

[8] Механические свойства редких металлов / Под ред. Л.Д. Соколова. М.: Металлургия, 1972. 288 с.

[9] Конструкционные материалы / Под ред. А.Т. Туманова. Т. 1. М.: Советская энциклопедия, 1965. 416 с.

[10] Буркхардт A. Механические и технологические свойства чистых металлов. М.: Металлургиздат, 1941. 264 с.

[11] Фрижсман Я.Б. Механические свойства металлов. М.: Машиностроение, 1974. 574 с.

[12] Благородные металлы. Справочник / Под ред. Е.М. Савицкого. М.: Металлургия, 1984. 511 с.

[13] Портной К.И. Структура и свойства композиционных материалов. М.: Машиностроение, 1979. 256 с. 